\title{
Genotype X Environment Interaction and Stability Parameters of Genotypes for Different Traits in Bhindi [Abelmoschus esculentus (L.) Moench]
}

\author{
Ramesh K. Sharma ${ }^{1}$, Ravi S. Singh ${ }^{2}$, Arun Kumar ${ }^{3}$, \\ Ashok K. Singh ${ }^{4}$ and S.K. Choudhary ${ }^{5}$
}

${ }^{1}$ Department of Horticulture (Vegetables \& Floriculture), ${ }^{2}$ Department of Plant Breeding and Genetics, ${ }^{5}$ Department of Agronomy, Bihar Agriculture College (BAU), Sabour-813 210, Bihar, India

${ }^{3}$ Directorate of Planning, Bihar Agricultural University, Sabour, Bhagalpur- 813210 , Bihar, India

${ }^{4}$ Department of Agronomy, Narendra Deva University of Agriculture and Technology, Ayodhya, 224229 Uttar Pradesh, India

*Corresponding author

\begin{abstract}
Keywords
Abelmoschus esculentus, Bhindi, G X E interaction, Stability

Article Info

Accepted:

18 May 2019

Available Online:

10 June 2019 flowering, days to first harvest, number of fruits per plant, fruit length $(\mathrm{cm})$, fruit diameter $(\mathrm{cm})$, fruit weight $(\mathrm{g})$, fruit yield per plant $(\mathrm{kg})$ and fruit yield per plot $(\mathrm{kg})$. Since many of the plant economically important characters are quantitatively inherited and highly influenced by the environmental condition. Phenotypic variation results from complex of three variables viz., genetic, environmental, and genotype X environment (GXE) interaction, hence, the stability of the genotypes in the predictable and unpredictable environments is an important factor for realizing the maximum yield. Since, precise information was not available on stability of promising genotypes in Bhindi that can be relied upon. Therefore, the present investigation was done on estimation of stability parameters of the genotypes for different traits in Bhindi to find out the performance of different genotypes, nature and magnitude of variability present under different dates of sowing in different crop seasons (Spring-Summer and Kharif) and to observe the stability of performance of various promising genotypes under different environments in both the seasons. The results thus indicated that genotypes HRB-9-2, Pb-57, HOE-202, D-1-87-5, Pusa Sawani, 71-14, KS-312 and D-1-87-16 had higher potentialities over environments for producing high yield. The genotypes HRB-9-2, Pb57, HOE-202, D-1-87-5 and Pusa Sawani had average response and are highly stable for fruit yield per plant. These genotypes are likely to perform well in all the environments of both the seasons (Spring-Summer and Kharif season). Thus genotypes as identified in the present study can further be exploited for higher yield and also in breeding for superior and stable genotypes of Bhindi.
\end{abstract}

\section{A B S T R A C T}

The material for the study consisted of twenty genotypes of Bhindi, which were grown in a Randomized Block Design (RBD) with three replication under six environments (three different dates of sowing in Spring-summer and Kharif). The observations were recorded on five randomly selected plants for characters viz., plant height $(\mathrm{cm})$. no. of branches per plant, days to first 


\section{Introduction}

Bhindi or Okra Abelmoschus esculentus (L.) Moencoh (family Malvaceae) is an important annual vegetable crop grown in tropics and sub-tropics for its tender green fruits. In India, it is grown during summer as well as in rainy season. India is a major okra producing country in the world comprising of $71 \%$ of total area under okra (FAOSTAT, 2014) and the average productivity of Bhindi in India is 12.00 tonnes/ha. Developing stable varieties of this crop that suits a particular location or multi-location is utmost important for cultivation and yield. In this regard, understanding the genotype $\mathrm{x}$ environment $(\mathrm{G}$ $\mathrm{X} \mathrm{E}$ ) interaction is important to breeders. The $\mathrm{G} X \mathrm{E}$ interactions are usually present under all conditions in pure lines, hybrids, synthetics or any other material used for breeding which complicate crop improvement programmes. Therefore, the performance of a crop in more than one environment should be performed to identify genotypes based on high stability for various yield related traits across different environment (Jindal et al., 2008). The uses of varietal mixture than homogeneous or pure lines have been suggested as a means to reduce GXE (Jenson, 1952). Allard and Bradshaw (1964) suggested that the heterozygous and heterogeneous populations offer the best opportunity to produce the varieties which show small genotype-environmental interactions. They used the term individual buffering for individuals where the individual members of a population are well buffered such that each member of the population well adapted to a range of environments and population buffering.

For developing a phenotypically stable variety, the information on the extent of $\mathrm{G} x \mathrm{E}$ interaction for yield and other component traits is essential, as the estimate of such interaction measures the differences in response of genotype to changing environments. Stability of the genotypes in the predictable and unpredictable environments is an important factor for realizing the maximum yield. Phenotypically stable lies are particularly of great importance in a country like India where the environmental condition under which crop is grown differs from region to region and within the same region. This necessitates screening and identifying phenotypically stable genotypes which could perform more or less uniformly under different environmental conditions. Earlier, we studied on the aspect of correlation, genetic variability, heritability and genetic advance in Bhindi (Sharma et al., 2016; Sharma et al., 2017). Furthermore, in the present study an attempt was made to investigate the stability parameters of the genotypes for different traits in Bhindi to find out the performance of different genotypes under different dates of sowing and crop seasons (Spring-summer and Kharif).

\section{Materials and Methods}

The experiment was conducted in the Permanent experimental area of the Department of Horticulture (Vegetable \& Floriculture), Bihar Agricultural College, Sabour $\left[87^{\circ} 2^{\prime} 42^{\prime \prime} \mathrm{E}\right.$ and $25^{\circ} 15^{\prime} 40^{\prime} \mathrm{N}$; $46 \mathrm{~m}$ mean sea level] in the heart of the vast IndoGangetic plain of Norh India. The climate of this place is tropical to sub-tropical of slightly semi-arid nature and is characterized by very dry summer, moderate rainfall and very cold winter. December and January are usually the coldest month whereas May and June are the hottest months. The rainfall is mainly distributed from middle of June to middle of October. The distribution has also been erratic thereby adversely affecting the crop. Data recording the prevailing conditions recorded standard week wise to observe the variation in different parameters of weather during each 
and every environment (six environments) of both the seasons.

The material for the study consisted of twenty genotypes of Bhindi (Table 1), which were grown in a Randomized Block Design (RBD) with three replication under six environments (three different dates of sowing in each of the two seasons prevailing in two crop season namely Spring-summer and Kharif (Table 2). In spring-summer season, the plot size comprised of $1.8 \mathrm{~m}$ length and $1.5 \mathrm{~m}$ breadth with 20 plants in each plot at the spacing of $45 \mathrm{~cm}$ from row to row and $30 \mathrm{~cm}$ from plant to plant. Each treatment was allocated to individual plot with the help of random table. There were four rows having 5 plants in each row making 20 plants in a plot. The observations were recorded on five randomly selected plants for different quantitative characters viz., plant height $(\mathrm{cm})$. no. of branches per plant, days to first flowering, days to first harvest, number of fruits per plant, fruit length $(\mathrm{cm})$, fruit diameter $(\mathrm{cm})$, fruit weight $(\mathrm{g})$, fruit yield per plant $(\mathrm{kg})$ and fruit yield per plot $(\mathrm{kg})$. The experimental plot was ploughed and cross ploughed four times followed by planking. Organic manure in the form of well rotten farm yard manures (FYM) @ $250 \mathrm{q} \mathrm{ha}^{-1}$ was applied at the time of last ploughing.

The experimental data for various characters detailed in above was recorded and subjected to statistical analysis using suitable technique for different characters. The technique of analysis of various for randomised block design (RBD) was adopted, as suggested by Panse and Sukhatme (1985). The phenotypic and genotypic variance was calculated method as suggested by Comstock and Robinson (1952). Phenotypic and genotypic coefficients of variation were calculated according to formula suggested by Burton (1952). The stability analysis was done following the method suggested by Eberhart and Russel (1966). This model defined a stable variety which has unit regression coefficient (bi=1) and a minimum deviation from the regression $\left(\mathrm{S}_{2} \mathrm{~d}=0\right)$ and high mean yield. The stability analysis consisted of three steps: (i) Environment-wise analysis of variance, (ii) Pooled analysis of variance over all environments, and (iii) analysis for stability parameters. The stability analysis was carried out for those characters only in which the GXE interactions were found to be significant.

\section{Results and Discussion}

High yield and better quality are the slogans of the day. Various indigenous varieties give poor yield of low quality. It is therefore, worthwhile to identify more promising stable varieties over a wide range of environments and expecting hybrid vigour in bhindi. So the total harvest in terms of tonnage and nutrition per unit area and per unit time can be enhanced in this short duration vegetable where individual plant carry more significance.

The information on quantitative genetics has made a major contribution synthesis of more efficient genotypes. Since many of the plant characters, which are of economic values, are quantitatively inherited and highly influenced by the environmental condition. It is difficult to judge whether observed variation is heritable or due to the influence of environments. Therefore, there is imperative need of partitioning the observed variability through into its heritable and non-heritable components through suitable genetic parameters viz., phenotypic and genotypic coefficient of variations, heritability and genetic advance for selection of a few promising genotypes from existing populations. Moreover, phenotypic variation is a complex of three variables viz., genetic, environmental and $\mathrm{G} X \mathrm{E}$ interaction. It is a common 
practice in trials involving varieties and breeding lines to grow a series of genotypes in a range of different environments. If all the genotypes respond similarly to all the environments, tested, their relative performance in other environments may be predicted with some confidence. A G X E interaction exists where the relative performance of genotypes changes from environment to environment. The presence of $\mathrm{G} X \mathrm{E}$ interaction is a major problem in getting reliable estimates of heritability and it makes it difficult to predict with greater accuracy the rate of genetic progress under selection for a given character.

The present study showed that the magnitude of mean performance of different traits including yield was more in all the environments (D4 TO D6) of the Kharif season as compared to that of Spring-summer season. In Table 3 range and mean of various plant characters in twenty genotypes of Bhindi grown under six environments is presented. Only one trait i.e., days to flowering did not follow this trend in D1 (earliest date of sowing) of the spring season. Here, we are showing the mean performance of Bhindi genotypes for one character only i.e. for fruit yield for per plant $(\mathrm{kg})$ tested in six environments due to space limitation for article (Table 4).

Phenotypic stability of component traits contributing to fruit yield stability was reported in Bhindi (Poshiya and Vashi, 1997; Kachhadia et al., 2011; Javia, 2014). Stability estimated to assess the stability over the environments was reported by More et al., (2018) in Bhindi, they found two genotypes, IC - 111493 and Arka Anamika were stable as they were flowered earlier and exhibited unit regression coefficient along with nonsignificant value of deviation from regression. In the present study linear regression (bi) has been considered as a measure of response of a particular genotype (Paroda and Hayes, 1971), whereas deviation around the regression line is considered as a measure of stability. Genotypes with non-significant deviation $\left(S^{2} d\right)$ along with high mean performance, average response are considered to be the most stable genotype. The pooled analysis of variance indicated that the $\mathrm{G} X \mathrm{E}$ interactions were highly significant for all the characters except days to flowering, days to first harvest and fruit length and thus, they were excluded from stability studies (Table 5). Remaining characters were subjected to stability analysis. Both linear as well as nonlinear components of GXE interactions were significant for the characters which were included for stability purposes (Table 6). The stability parameters of plant height, number of branches per plant, number of fruits per plant, fruit diameter, fruit weight and fruit yield per plant have been presented in (Table 7).

The stability parameters for plant height revealed that the genotypes $\mathrm{Sel} .7$ and $\mathrm{Pb}-57$ were highly stable for this trait over all the environments as they had mean value above the population mean, regression co-efficient (bi) near to one and non-significant deviation from regression ( $\mathrm{S}^{2} \mathrm{~d}$ close to zero). HRB-9-2, HOE-202, Pb-57, HRB-55 and Pusa Sawani were found stable for number of branches per plant as they had mean value above the population mean, average response (bi near to unity) and low value of deviation from regression ( $\mathrm{S}^{2} \mathrm{~d}$ near to zero). HRB-9-2, Pb57, HOE-202, D-1-87-5, Sel.-4 and HRB-55 were found to be stable in respect of number of fruits per plant suggesting thereby better performance of these genotypes under all the environments.

Both linear as well as non-linear components of G X E interactions for fruit yield per plant were found to be significant suggesting that genotypes differed significantly in their response to different environments (Table 6). 
Table.1 List of genotypes with source included in the experiment

\begin{tabular}{|l|l|l|l|}
\hline Sl.No. & Genotypes & Source & Symbol \\
\hline $\mathbf{1}$ & AROH-1 & Ankur seed & G1 \\
\hline $\mathbf{2}$ & B.O.-1 & O.A.U., Bhubneshwer & G2 \\
\hline $\mathbf{3}$ & Sel.-2 & NBPGR, Delhi & G3 \\
\hline $\mathbf{4 .}$ & Vaishali Vadhu & B.A.C., Sabour & G4 \\
\hline $\mathbf{5}$ & D-1-87-16 & B.A.C., Sabour & G5 \\
\hline $\mathbf{6}$ & Sel.-7 & IIHR, Banglore & G6 \\
\hline $\mathbf{7}$ & HOE-301 & Hoechst & G7 \\
\hline $\mathbf{8}$ & Sel.-4 & IIHT, Banglore & G8 \\
\hline $\mathbf{9}$ & HRB-55 & H.A.U., Hissar & G9 \\
\hline $\mathbf{1 0}$ & B.O.-2 & O.A.U. Bhubneshwer & G10 \\
\hline $\mathbf{1 1}$ & Pb-57 & Parbhani & G11 \\
\hline $\mathbf{1 2}$ & Sel.10 & IIHR, Banglore & G12 \\
\hline $\mathbf{1 3}$ & KS-312 & Kalyanpur & G13 \\
\hline $\mathbf{1 4}$ & HOE-202 & Hoechst & G14 \\
\hline $\mathbf{1 5}$ & 71-14 & B.A.C., Sabour & G15 \\
\hline $\mathbf{1 6}$ & D-1-87-5 & B.A.C., Sabour & G16 \\
\hline $\mathbf{1 7}$ & N.D.O.-25 & N.D.A.U.T., Faizabad & G17 \\
\hline $\mathbf{1 8}$ & HRB-9-2 & HAU, Hissar & G18 \\
\hline $\mathbf{1 9}$ & Sel.-8 & IIHR, Banglore & G19 \\
\hline $\mathbf{2 0}$ & Pusa Sawani & IARI, Delhi & G20 \\
\hline
\end{tabular}

Table.2 the details of six environments under which experiments were conducted

\begin{tabular}{|l|l|l|c|}
\hline Sl.No. & Seasons & Different environments & Symbols \\
\hline \multirow{2}{*}{$\mathbf{3}$} & Spring-Summer & $18^{\text {th }}$ January & D1 \\
\cline { 3 - 4 } & & $7^{\text {th }}$ February & D2 \\
\cline { 3 - 4 } & & $27^{\text {th }}$ February & D3 \\
\hline \multirow{2}{*}{$\mathbf{2}$} & \multirow{2}{*}{ Kharif } & $10^{\text {th }}$ June & D4 \\
\cline { 3 - 4 } & & $30^{\text {th }}$ June & D5 \\
\cline { 3 - 4 } & & $20^{\text {th }}$ July & D6 \\
\hline
\end{tabular}

Table.3 Range and mean of various plant characters in twenty genotypes of Bhindi grown under six environments

\begin{tabular}{|c|c|c|c|c|}
\hline Characters & Environment & Range & Mean & \pm S.E.(m) \\
\hline \multirow[t]{6}{*}{ 1. Plant height $(\mathrm{cm})$. } & $\mathrm{D}_{1}$ & $49.08-71.30$ & 59.27 & \pm 2.7731 \\
\hline & $\mathrm{D}_{2}$ & $62.23-93.27$ & 76.88 & \pm 2.7731 \\
\hline & $\mathrm{D}_{3}$ & 59. $72-90.26$ & 76.56 & \pm 2.6424 \\
\hline & $\mathrm{D}_{4}$ & $96.32-151.26$ & 121.52 & \pm 6.9616 \\
\hline & $D_{5}$ & $87.31-136.00$ & 109.54 & +5.0302 \\
\hline & $D_{6}$ & $82.25-128.75$ & 103.34 & $\begin{array}{l}+5.3909 \\
\end{array}$ \\
\hline \multirow{4}{*}{$\begin{array}{l}\text { 2. } \begin{array}{l}\text { Number of } \\
\text { Branches plant }^{-1}\end{array}\end{array}$} & $\mathrm{D}_{1}$ & $2.07-3.93$ & 3.15 & +0.1732 \\
\hline & $\mathrm{D}_{2}$ & $2.27-4.27$ & 3.55 & \pm 0.1218 \\
\hline & $D_{3}$ & $2.07-4.20$ & 3.53 & \pm 0.1953 \\
\hline & $\mathrm{D}_{4}$ & $4.00-9.20$ & 6.29 & \pm 0.3637 \\
\hline
\end{tabular}




\begin{tabular}{|c|c|c|c|c|}
\hline & $\mathrm{D}_{5}$ & $3.87-8.93$ & 5.84 & \pm 0.3619 \\
\hline & $\mathrm{D}_{6}$ & $3.67-8.27$ & 5.31 & +0.3784 \\
\hline \multirow[t]{6}{*}{ 3. Days to flow ering } & $D_{1}$ & $47.00-50.33$ & 48.08 & \pm 1.5281 \\
\hline & $\mathrm{D}_{2}$ & $32.33-36.0$ & 33.77 & \pm 1.0210 \\
\hline & $\mathrm{D}_{3}$ & $32.33-36.33$ & 34.16 & \pm 1.2384 \\
\hline & $\mathrm{D}_{4}$ & $35.67-39.00$ & 37.10 & \pm 1.3627 \\
\hline & $\mathrm{D}_{5}$ & $35.33-40.33$ & 37.55 & +1.3609 \\
\hline & $\mathrm{D}_{6}$ & $35.33-40.67$ & 37.92 & \pm 1.4537 \\
\hline \multirow{6}{*}{$\begin{array}{l}\text { 4. Days to first } \\
\text { harvest }\end{array}$} & $D_{1}$ & $54.33-57.33$ & 56.23 & \pm 1.6675 \\
\hline & $\mathrm{D}_{2}$ & $39.00-43.00$ & 41.13 & \pm 1.5826 \\
\hline & $\mathrm{D}_{3}$ & $39.67-42.67$ & 41.30 & \pm 1.2911 \\
\hline & $\mathrm{D}_{4}$ & $42.33-46.00$ & 44.17 & \pm 1.4556 \\
\hline & $D_{5}$ & $42.00-47.67$ & 45.08 & \pm 1.4092 \\
\hline & $D_{6}$ & $42.33-47.67$ & 45.37 & +1.7337 \\
\hline \multirow{6}{*}{$\begin{array}{l}\text { 5. Number of fruit } \\
\text { plant }^{-1}\end{array}$} & $D_{1}$ & $4.67-10.33$ & 6.94 & \pm 0.4656 \\
\hline & $\mathrm{D}_{2}$ & $5.33-13.33$ & 8.32 & \pm 0.4450 \\
\hline & $\mathrm{D}_{3}$ & $5.33-13.33$ & 8.27 & \pm 0.5004 \\
\hline & $\mathrm{D}_{4}$ & $11.67-26.67$ & 17.03 & \pm 1.0531 \\
\hline & $D_{5}$ & $10.67-24.33$ & 15.05 & +0.8505 \\
\hline & $\mathrm{D}_{6}$ & $10.00-21.67$ & 13.61 & \pm 0.7457 \\
\hline \multirow[t]{6}{*}{ 6. Fruit length $(\mathrm{cm})$} & $\mathrm{D}_{1}$ & $9.36-13.76$ & 11.23 & \pm 0.5970 \\
\hline & $\mathrm{D}_{2}$ & $9.64-13.98$ & 11.67 & \pm 0.5057 \\
\hline & $\mathrm{D}_{3}$ & $9.54-13.74$ & 11.50 & \pm 0.4837 \\
\hline & $\mathrm{D}_{4}$ & $11.50-15.73$ & 13.35 & \pm 0.7701 \\
\hline & $D_{5}$ & $11.70-15.18$ & 13.35 & \pm 0.7460 \\
\hline & $D_{6}$ & $11.98-15.61$ & 13.40 & \pm 0.5682 \\
\hline \multirow{6}{*}{$\begin{array}{l}\text { 7. Fruit diameter } \\
(\mathrm{cm})\end{array}$} & $\mathrm{D}_{1}$ & $1.28-1.68$ & 1.49 & \pm 0.0582 \\
\hline & $D_{2}$ & $1.28-1.94$ & 1.66 & +0.0538 \\
\hline & $\mathrm{D}_{3}$ & $1.26-1.87$ & 1.55 & \pm 0.0681 \\
\hline & $\mathrm{D}_{4}$ & $1.35-2.28$ & 2.04 & \pm 0.0842 \\
\hline & $D_{5}$ & $1.68-2.34$ & 2.08 & +0.0838 \\
\hline & $\mathrm{D}_{6}$ & $1.59-2.13$ & 1.99 & \pm 0.0704 \\
\hline \multirow[t]{6}{*}{ 8. Fruit weight (kg) } & $\mathrm{D}_{1}$ & $8.25-14.26$ & 10.74 & \pm 0.4378 \\
\hline & $\mathrm{D}_{2}$ & $9.69-14.00$ & 11.77 & \pm 0.4735 \\
\hline & $\mathrm{D}_{3}$ & $9.72-14.24$ & 11.63 & \pm 0.4697 \\
\hline & $\mathrm{D}_{4}$ & $11.25-15.93$ & 13.86 & \pm 0.5237 \\
\hline & $D_{5}$ & $10.62-16.97$ & 13.90 & \pm 0.5419 \\
\hline & $\mathrm{D}_{6}$ & $10.93-15.80$ & 13.57 & \pm 0.5495 \\
\hline \multirow{6}{*}{$\begin{array}{l}\text { 9. Fruit yield plant } \\
\text { (kg) }\end{array}$} & $D_{1}$ & $0.055-0.106$ & 0.073 & \pm 0.0035 \\
\hline & $\mathrm{D}_{2}$ & $0.072-0.137$ & 0.096 & \pm 0.0043 \\
\hline & $\mathrm{D}_{3}$ & $0.072-0.135$ & 0.094 & \pm 0.0026 \\
\hline & $\mathrm{D}_{4}$ & $0.176-0.334$ & 0.332 & \pm 0.0107 \\
\hline & $D_{5}$ & $0.156-0.297$ & 0.260 & +0.0123 \\
\hline & $\mathrm{D}_{6}$ & $0.136-0.259$ & 0.183 & \pm 0.0090 \\
\hline \multirow{6}{*}{$\begin{array}{l}\text { 10. Fruit yield plot } \\
\text { (kg) }\end{array}$} & $\mathrm{D}_{1}$ & $1.10-2.03$ & 1.45 & \pm 0.0678 \\
\hline & $\mathrm{D}_{2}$ & $1.43-2.73$ & 1.91 & \pm 0.1152 \\
\hline & $\mathrm{D}_{3}$ & $1.42-2.70$ & 1.88 & \pm 0.0889 \\
\hline & $\mathrm{D}_{4}$ & $3.51-6.69$ & 4.64 & +0.2948 \\
\hline & $D_{5}$ & $3.13-5.94$ & 4.13 & +0.1816 \\
\hline & $D_{6}$ & $2.74-5.18$ & 3.66 & \pm 0.1495 \\
\hline
\end{tabular}


Table.4 Mean performance of Bhindi genotypes for fruit yield for per plant $(\mathrm{kg})$ tested in six environments

\begin{tabular}{|c|c|c|c|c|c|c|c|}
\hline \multirow[t]{2}{*}{ Sl. no. } & \multirow[t]{2}{*}{ Genotypes } & \multicolumn{3}{|c|}{ Spring - Summer } & \multicolumn{2}{|c|}{ I Kharif Season } & \multirow[b]{2}{*}{$\mathrm{D}_{6}$} \\
\hline & & $\mathrm{D}_{1}$ & $\mathrm{D}_{2}$ & $\mathrm{D}_{3}$ & $\mathrm{D}_{4}$ & $\mathrm{D}_{5}$ & \\
\hline 1. & AROH-1 & 0.062 & 0.081 & 0.082 & 0.202 & 0.178 & 0.163 \\
\hline 2. & B.O.- 1 & 0.066 & 0.087 & 0.084 & 0.212 & 0.184 & 0.171 \\
\hline 3. & Sel.-2 & 0.065 & 0.086 & 0.084 & 0.206 & 0.179 & 0.165 \\
\hline 4. & Vaishali Vadhu & $0 . .069$ & 0.094 & 0.091 & 0.227 & 0.202 & 0.180 \\
\hline 5. & D-1-87-16 & 0.075 & 0.098 & 0.095 & 0.236 & 0.211 & 0.187 \\
\hline 6. & Shail.-7 & 0.068 & 0.091 & 0.093 & 0.217 & 0.189 & 0.169 \\
\hline 7. & Hoe-301 & 0.073 & 0.098 & 0.088 & 0.231 & 0.196 & 0.183 \\
\hline 8. & Shail.-4 & 0.068 & 0.090 & 0.090 & 0.221 & 0.195 & 0.171 \\
\hline 9. & HRB-55 & 0.066 & 0.084 & 0.081 & 0.210 & 0.181 & 0.160 \\
\hline 10. & B.O.- 2 & 0.055 & 0.072 & 0.071 & 0.176 & 0.156 & 0.136 \\
\hline 11. & $\mathrm{~Pb}-57$ & 0.092 & 0.122 & 0.120 & 0.303 & 0.264 & 0.234 \\
\hline 12. & Shail.-10 & 0.064 & 0.083 & 0.084 & 0.198 & 0.177 & 0.153 \\
\hline 13. & KS-312 & 0.070 & 0.096 & 0.093 & 0.235 & 0.210 & 0.104 \\
\hline 14. & HOE-202 & 0.086 & 0.113 & 0.109 & 0.279 & 0.251 & 0.212 \\
\hline 15. & $71-14$ & 0.076 & 0.099 & 0.102 & 0.239 & 0.215 & 0.102 \\
\hline 16. & D-1-87-5 & 0.085 & 0.110 & 0.109 & 0.259 & 0.220 & 0.203 \\
\hline 17. & N.D.O-25 & 0.061 & 0.078 & 0.079 & 0.189 & 0.164 & 0.146 \\
\hline 18. & HRB-9-2 & 0.106 & 0.137 & 0.135 & 0.334 & 0.297 & 0.259 \\
\hline 19. & Shail.-8 & 0.072 & 0.093 & 0.093 & 0.225 & 0.203 & 0.179 \\
\hline \multirow[t]{4}{*}{20.} & Pusa Sawani & 0.077 & 0.104 & 0.102 & 0.248 & 0.251 & 0.219 \\
\hline & Mean & 0.073 & 0.096 & 0.094 & 0.232 & 0.207 & 0.183 \\
\hline & C.D. at $5 \%$ & 0.0101 & 0.0124 & 0.0075 & 0.0305 & 0.0312 & 0.0257 \\
\hline & C.V. (\%) & 8.37 & 7.84 & 4.81 & 7.95 & 10.31 & 8.50 \\
\hline
\end{tabular}

Where, $D_{1}=18^{\text {th }}$ January $\quad D_{2}=7^{\text {th }}$ February $\quad D_{3}=27^{\text {th }}$ February $\quad D_{4}=10^{\text {th }}$ June $\quad D_{5}=30^{\text {th }}$ June $\quad D_{6}=20^{\text {th }}$ Jul

Table.5 Pooled analysis of variance (mean square) of various characters of bhindi genotypes under study in six environments

\begin{tabular}{|l|l|l|l|l|l|l|l|l|l|l|}
\hline $\begin{array}{l}\text { Source of } \\
\text { Variation }\end{array}$ & d.f. & $\begin{array}{l}\text { Plant } \\
\text { Height } \\
\text { (Cm) }\end{array}$ & $\begin{array}{l}\text { Number } \\
\text { of } \\
\text { Branches } \\
\text { Per Plant }\end{array}$ & $\begin{array}{l}\text { Days to } \\
\text { Flowering }\end{array}$ & $\begin{array}{l}\text { Days to } \\
\text { Frist } \\
\text { Harvest }\end{array}$ & $\begin{array}{l}\text { No. of } \\
\text { Fruits Per } \\
\text { Plant }\end{array}$ & $\begin{array}{l}\text { Fruit } \\
\text { Length } \\
\text { (Cm) }\end{array}$ & $\begin{array}{l}\text { Fruit } \\
\text { Diamet } \\
\text { er } \\
\text { (Cm) }\end{array}$ & $\begin{array}{l}\text { Fruit } \\
\text { Weight } \\
\text { (g) }\end{array}$ & $\begin{array}{l}\text { Fruit Yield } \\
\text { Per Plant } \\
(\mathbf{K g})\end{array}$ \\
\hline Environment & 5 & $34122.0782^{* *}$ & $110.6443^{* *}$ & $1623.4244^{* *}$ & 1846.4161 & $1064.1244^{* *}$ & $66.3579^{* *}$ & $4.4204^{* *}$ & $111.3599^{* * *}$ & $0.27648^{* *}$ \\
\hline Genotype & 19 & $1231.2809^{* *}$ & $10.1352^{* *}$ & 6.5094 & 8.5923 & $102.2959^{* *}$ & $20.6265^{* *}$ & $0.1881^{* *}$ & $26.2386^{* *}$ & $0.01063^{* *}$ \\
\hline $\begin{array}{l}\text { Genotype X } \\
\text { Environment }\end{array}$ & 95 & $369.4533^{* *}$ & $1.3961^{* *}$ & $3.5999 \mathrm{NS}$ & $3.0231 \mathrm{NS}$ & $3.8777^{* *}$ & $1.0932 \mathrm{NS}$ & $0.0412^{* *}$ & $2.8766^{* *}$ & $0.00044^{* *}$ \\
\hline Pooled Error & 240 & 65.4593 & 0.2509 & 5.2000 & 6.8528 & 1.5778 & 1.1503 & 0.0160 & 1.2485 & 0.00019 \\
\hline Total & 359 & & & & & & & & & \\
\hline
\end{tabular}

$*$ and ${ }^{* *}$ Significant at $5 \%$ and $1 \%$ probability, respectively 
Table.6 ANOVA for stability parameters of Bhindi genotypes tested in six environments

\begin{tabular}{|l|l|l|l|l|l|l|l|l|}
\hline & \multicolumn{7}{|c|}{ MEAN SUM OF SQUARES } \\
\hline $\begin{array}{l}\text { Source of } \\
\text { Variation }\end{array}$ & d.f. & $\begin{array}{l}\text { Plant } \\
\text { Height } \\
(\mathrm{Cm})\end{array}$ & $\begin{array}{l}\text { Number of } \\
\text { Branches } \\
\text { Per Plant }\end{array}$ & $\begin{array}{l}\text { Number } \\
\text { Fruits } \\
\text { Per Plant }\end{array}$ & $\begin{array}{l}\text { Fruits } \\
\text { Diamet } \\
\text { er }(\mathrm{Cm})\end{array}$ & $\begin{array}{l}\text { Fruits } \\
\text { Weight } \\
(\mathrm{g})\end{array}$ & $\begin{array}{l}\text { Fruit Yield } \\
\text { Per Plant } \\
(\mathrm{Kg})\end{array}$ & $\begin{array}{l}\text { Fruit Yield } \\
\text { Per plot } \\
(\mathrm{Kg})\end{array}$ \\
\hline Total & 119 & & & & & & & \\
\hline Genotype (G) & 19 & $425.1775^{* *}$ & $3.3743^{* *}$ & $34.096^{* *}$ & $0.0657^{* *}$ & $8.7532^{* *}$ & $0.003533^{* *}$ & $1.4083^{* *}$ \\
\hline Environment+(GXE) & 100 & $682.8923^{* *}$ & $2.2858^{* *}$ & $1.8989^{* *}$ & $0.0800^{* *}$ & $2.7693^{* *}$ & $0.004745^{* *}$ & $1.9045^{* *}$ \\
\hline Environment(Linear) & 1 & $56870.3128^{* *}$ & $184.4142^{* *}$ & $1774.0423^{* *}$ & $7.0680^{* *}$ & $185.7648^{* *}$ & $0.460330^{* *}$ & $184.6721^{* *}$ \\
\hline G X E (Linear) & 19 & $516.6460^{* *}$ & $2.0539^{* *}$ & $4.9144^{* *}$ & $0.0399^{* *}$ & $3.2608^{* *}$ & $0.000684^{* *}$ & $0.2824^{* *}$ \\
\hline Pooled Deviation & 80 & 20.0330 & 0.0643 & 0.3688 & $0.0122++$ & 0.3651 & 0.000014 & 0.0052 \\
\hline Pooled Error & 240 & 21.8198 & 0.0850 & 0.4849 & 0.0053 & 0.4161 & 0.000078 & 0.0286 \\
\hline
\end{tabular}

*and $* *$ Significant at 5 and 1 per cent probability level, respectively, when tested against pooled deviation. ++

Significant at $1 \%$ probability level, when tested against pooled error.

Table.7a Stability parameters of Bhindi genotypes for plant height $(\mathrm{cm})$ and number of branches per plant under study tested in six environments

\begin{tabular}{|c|c|c|c|c|c|c|c|}
\hline \multirow[t]{2}{*}{ Sl.no. } & \multirow[t]{2}{*}{$\begin{array}{l}\text { Bhindi } \\
\text { Genotypes }\end{array}$} & \multicolumn{3}{|c|}{ Plant Height (cm.) } & \multicolumn{3}{|c|}{$\begin{array}{l}\text { Number of Branches per } \\
\text { Plant }\end{array}$} \\
\hline & & $X$ & bi & $S^{2} d$ & $\mathrm{X}$ & bi & $S^{2} d$ \\
\hline 1. & AROH-1 & 92.08 & 1.4923 & 15.08 & 3.47 & 1.0249 & -0.03 \\
\hline 2. & B.O.-1 & 95.00 & 1.5337 & 16.02 & 4.59 & 1.3657 & -0.05 \\
\hline 3. & Sel-2 & 84.22 & 1.2725 & 2.93 & 3.91 & 1.1242 & -0.02 \\
\hline 4. & VaishaliVadhu & 84.09 & 0.7828 & -15.72 & 4.46 & 1.3001 & 0.07 \\
\hline 5. & D-1-87-16 & 81.24 & 0.7812 & -21.11 & 4.29 & 0.6725 & -0.02 \\
\hline 6. & Sel.-7 & 103.61 & 1.0727 & -19.84 & 6.39 & 1.9369 & 0.11 \\
\hline 7. & HOE-301 & 92.41 & 1.3317 & -5.76 & 5.07 & 1.4538 & -0.06 \\
\hline 8. & Sel.-4 & 89.01 & 0.9984 & -20.64 & 5.30 & 1.6660 & 0.02 \\
\hline 9. & HRB-55 & 84.83 & 0.5210 & 6.01 & 4.80 & 0.9772 & -0.04 \\
\hline 10. & B.O.-2 & 105.91 & 1.3567 & -13.87 & 5.59 & 1.4709 & -0.02 \\
\hline 11. & $\mathrm{~Pb}-57$ & 93.70 & 0.7603 & -2.70 & 5.16 & 1.0481 & -0.07 \\
\hline 12. & Sel.-10 & 99.93 & 1.7996 & $38.38^{*}$ & 3.81 & 0.8161 & -0.06 \\
\hline 13. & KS-312 & 88.91 & 0.3051 & $35.84 *$ & 4.18 & 0.2738 & 0.00 \\
\hline 14. & HOE-202 & 106.00 & 1.3386 & -17.83 & 5.18 & 1.0744 & -0.05 \\
\hline 15. & $71-14$ & 86.63 & 0.2817 & $33.97 *$ & 4.38 & 0.3744 & -0.01 \\
\hline 16. & D-1-87-5 & 97.16 & 1.4012 & -13.45 & 4.24 & 0.7563 & -0.03 \\
\hline 17. & N.D.O. -25 & 77.03 & 0.6038 & -16.35 & 3.99 & 0.2952 & 0.03 \\
\hline 18. & HRB-9-2 & 92.18 & 0.6898 & -6.08 & 5.27 & 1.1476 & -0.06 \\
\hline 19. & Sel.-8 & 78.91 & 0.8933 & -30.90 & 3.40 & 0.3718 & -0.08 \\
\hline \multirow[t]{3}{*}{20.} & PusaSawani & 91.10 & 0.7835 & -10.11 & 4.75 & 0.8501 & -0.03 \\
\hline & Mean & 91.19 & 1.0000 & & 4.61 & 1.0000 & \\
\hline & S.E. m+ & 2.00 & 0.0839 & & 0.11 & 0.0835 & \\
\hline
\end{tabular}


Table.7b Stability parameters of Bhindi genotypes for no. of fruits per plant and fruit Diameter $(\mathrm{cm})$ under study tested in six environments

\begin{tabular}{|c|c|c|c|c|c|c|c|}
\hline \multirow[t]{2}{*}{ Sl.no. } & \multirow[t]{2}{*}{ Bhindi genotypes } & \multicolumn{3}{|c|}{ No. of Fruits per Plant } & \multicolumn{3}{|c|}{ Fruit Diameter (cm) } \\
\hline & & $\mathrm{X}$ & bi & $S^{2} d$ & $\mathrm{X}$ & bi & $S^{2} d$ \\
\hline 1. & AROH-1 & 9.11 & 0.8389 & -0.4756 & 1.73 & 1.3397 & $0.0108 *$ \\
\hline 2. & B.O.-1 & 10.00 & 0.9757 & -0.3177 & 1.81 & 1.3548 & 0.0007 \\
\hline 3. & Sel-2 & 9.33 & 0.8423 & -0.4512 & 1.97 & 0.7206 & 0.0016 \\
\hline 4. & VaishaliVadhu & 11.28 & 1.2102 & 0.0072 & 1.88 & 1.1518 & 0.0045 \\
\hline 5. & D-1-87-16 & 11.00 & 1.2816 & 0.9235 & 1.71 & 0.7887 & $0.0833 * *$ \\
\hline 6. & Sel.-7 & 10.39 & 0.9734 & -0.2005 & 1.81 & 1.0469 & -0.0015 \\
\hline 7. & HOE-301 & 12.33 & 1.2836 & -0.4285 & 1.90 & 0.6234 & -0.0039 \\
\hline 8. & Sel.-4 & 12.39 & 1.1004 & -0.3564 & 1.84 & 1.4037 & 0.0029 \\
\hline 9. & HRB-55 & 12.00 & 1.0250 & 0.1709 & 1.86 & 1.1764 & 0.0018 \\
\hline 10. & B.O.-2 & 7.95 & 1.0465 & -0.3609 & 1.90 & 0.8880 & $0.0165 * *$ \\
\hline 11. & $\mathrm{~Pb}-57$ & 14.50 & 1.0561 & -0.3937 & 1.89 & 1.0284 & -0.0013 \\
\hline 12. & Sel.-10 & 11.61 & 1.0905 & 0.4140 & 1.92 & 1.0694 & -0.0053 \\
\hline 13. & KS-312 & 11.67 & 0.6665 & -0.3285 & 1.68 & 1.1514 & $0.0369 * *$ \\
\hline 14. & HOE-202 & 13.95 & 1.0771 & -0.4593 & 1.81 & 0.9926 & -0.0037 \\
\hline 15. & $71-14$ & 10.22 & 0.8167 & 0.2273 & 1.74 & 1.4817 & 0.0009 \\
\hline 16. & D-1-87-5 & 13.67 & 1.1284 & -0.4186 & 1.57 & 0.4938 & 0.0063 \\
\hline 17. & N.D.O.-25 & 8.72 & 0.7972 & -0.3881 & 1.86 & 0.8829 & -0.0019 \\
\hline 18. & HRB-9-2 & 18.28 & 1.0095 & -0.2577 & 1.68 & 1.0710 & -0.0051 \\
\hline 19. & Sel.-8 & 9.78 & 0.8791 & -0.3094 & 1.84 & 0.8016 & -0.0014 \\
\hline \multirow[t]{3}{*}{20.} & PusaSawani & 12.72 & 0.9014 & 1.0808 & 1.65 & 0.5333 & -0.0026 \\
\hline & Mean & 11.54 & 1.0000 & & 1.80 & 1.0000 & \\
\hline & S.E. $m \pm$ & 0.27 & 0.0645 & & 0.05 & 0.1858 & \\
\hline
\end{tabular}

Table.7c Stability parameters of Bhindi genotypes for fruit weight $(\mathrm{g})$ and fruit yield per plant $(\mathrm{Kg})$ under study tested in six environments

\begin{tabular}{|c|c|c|c|c|c|c|c|}
\hline \multirow[t]{2}{*}{ Sl.no. } & \multirow[t]{2}{*}{ Bhindi Genotypes } & \multicolumn{3}{|c|}{ Fruit Weight (g) } & \multicolumn{3}{|c|}{ Fruit Yield Per Plant (kg) } \\
\hline & & $\mathrm{X}$ & bi & $S^{2} d$ & $\mathrm{X}$ & bi & $S^{2} d$ \\
\hline 1. & AROH-1 & 13.64 & 0.9929 & -0.373 & 0.128 & 1.0810 & -0.000072 \\
\hline 2. & B.O.-1 & 13.14 & 0.5444 & -0.246 & 0.134 & 0.9145 & -0.000068 \\
\hline 3. & Sel-2 & 13.65 & 0.9601 & -0.150 & 0.131 & 0.8758 & -0.000072 \\
\hline 4. & VaishaliVadhu & 12.71 & 0.0974 & -0.189 & 0.144 & 0.9883 & -0.000077 \\
\hline 5. & D-1-87-16 & 13.99 & 0.5826 & 0.241 & 0.150 & 1.3178 & -0.000077 \\
\hline 6. & Sel.-7 & 12.73 & 1.7774 & -0.046 & 0.138 & 0.9065 & -0.000071 \\
\hline 7. & HOE-301 & 11.64 & 0.5426 & -0.185 & 0.145 & 0.9773 & -0.000052 \\
\hline 8. & Sel.-4 & 10.89 & 0.8961 & -0.264 & 0.139 & 0.9489 & -0.000076 \\
\hline 9. & HRB-55 & 10.50 & 1.0813 & -0.230 & 0.130 & 0.8962 & -0.000070 \\
\hline 10. & B.O.-2 & 13.65 & 0.7453 & -0.052 & 0.111 & 1.1553 & -0.000077 \\
\hline 11. & $\mathrm{~Pb}-57$ & 12.48 & 1.0053 & -0.373 & 0.189 & 1.0066 & -0.000074 \\
\hline 12. & Sel.-10 & 10.74 & 0.4855 & 0.479 & 0.127 & 0.8327 & -0.000074 \\
\hline 13. & KS-312 & 12.09 & 2.3053 & -0.320 & 0.148 & 1.0322 & -0.000077 \\
\hline 14. & HOE-202 & 12.04 & 1.4681 & -0.359 & 0.175 & 1.0159 & -0.000063 \\
\hline 15. & $71-14$ & 14.48 & 1.1725 & 1.737 & 0.152 & 1.0097 & -0.000063 \\
\hline 16. & D-1-87-5 & 11.76 & 0.9457 & -0.358 & 0.166 & 1.0792 & -0.000077 \\
\hline 17. & N.D.O.-25 & 13.43 & 0.5945 & -0.075 & 0.120 & 0.7878 & -0.000074 \\
\hline 18. & HRB-9-2 & 11.23 & 0.9808 & -0.283 & 0.211 & 1.0305 & -0.000075 \\
\hline 19. & Sel.-8 & 14.35 & 1.1480 & 0.156 & 0.144 & 0.9699 & -0.000076 \\
\hline \multirow[t]{3}{*}{20.} & PusaSawani & 12.48 & 1.6747 & -0.130 & 0.165 & 1.1779 & -0.000094 \\
\hline & Mean & 12.58 & & & 0.147 & 1.0000 & \\
\hline & S.E. $m \pm$ & 0.2702 & & & 0.0017 & 0.0247 & \\
\hline
\end{tabular}

\footnotetext{
$*$ and $* *$ Significant at $5 \%$ and $1 \%$, respectively
} 
Table.8 List of genotypes with high yield performance and high stability along with the stability of different quantitative characters under study

\begin{tabular}{|c|c|c|c|c|c|c|}
\hline \multirow{2}{*}{$\begin{array}{l}\text { Stable } \\
\text { Genotypes with } \\
\text { high mean yield }\end{array}$} & \multicolumn{6}{|c|}{ Stability of Different quantitative Characters } \\
\hline & $\begin{array}{l}\text { Plant height } \\
\text { (cm) }\end{array}$ & $\begin{array}{l}\text { Number of } \\
\text { branches per } \\
\text { plant }\end{array}$ & $\begin{array}{l}\text { Number of } \\
\text { fruits per plant }\end{array}$ & $\begin{array}{l}\text { Fruit } \\
\text { diameter } \\
(\mathrm{cm})\end{array}$ & $\begin{array}{l}\text { Fruit weight } \\
\text { (g) }\end{array}$ & $\begin{array}{l}\text { Fruit yield per } \\
\text { plant } \\
(\mathrm{kg})\end{array}$ \\
\hline 1.HRB-9-2 & $\begin{array}{l}\text { Unstable, } \\
\text { Above } \\
\text { Population } \\
\text { Mean }\end{array}$ & $\begin{array}{c}\text { stable, } \\
\text { Above } \\
\text { Population } \\
\text { Mean }\end{array}$ & $\begin{array}{l}\text { stable, } \\
\text { Above } \\
\text { Population } \\
\text { Mean }\end{array}$ & $\begin{array}{l}\text { Unstable, } \\
\text { Above } \\
\text { Population } \\
\text { Mean }\end{array}$ & $\begin{array}{l}\text { Unstable, } \\
\text { Above } \\
\text { Population } \\
\text { Mean }\end{array}$ & $\begin{array}{c}\text { stable, } \\
\text { Above } \\
\text { Population } \\
\text { Mean }\end{array}$ \\
\hline 2. $p b-57$ & $\begin{array}{l}\text { stable, } \\
\text { Above } \\
\text { Population } \\
\text { Mean }\end{array}$ & $\begin{array}{l}\text { stable, } \\
\text { Above } \\
\text { Population } \\
\text { Mean }\end{array}$ & $\begin{array}{l}\text { stable, } \\
\text { Above } \\
\text { Population } \\
\text { Mean }\end{array}$ & $\begin{array}{l}\text { stable, } \\
\text { Above } \\
\text { Population } \\
\text { Mean }\end{array}$ & $\begin{array}{l}\text { Unstable, } \\
\text { Above } \\
\text { Population } \\
\text { Mean }\end{array}$ & $\begin{array}{l}\text { stable, } \\
\text { Above } \\
\text { Population } \\
\text { Mean }\end{array}$ \\
\hline 3. HOE-202 & $\begin{array}{l}\text { Unstable, } \\
\text { Above } \\
\text { Population } \\
\text { Mean }\end{array}$ & $\begin{array}{l}\text { stable, } \\
\text { Above } \\
\text { Population } \\
\text { Mean }\end{array}$ & $\begin{array}{l}\text { stable, } \\
\text { Above } \\
\text { Population } \\
\text { Mean }\end{array}$ & $\begin{array}{c}\text { Stable, } \\
\text { Above } \\
\text { Population } \\
\text { Mean }\end{array}$ & $\begin{array}{l}\text { Unstable, } \\
\text { Above } \\
\text { Population } \\
\text { Mean }\end{array}$ & $\begin{array}{l}\text { stable, } \\
\text { Above } \\
\text { Population } \\
\text { Mean }\end{array}$ \\
\hline 4. D-1-87-5 & $\begin{array}{l}\text { Unstable, } \\
\text { Above } \\
\text { Population } \\
\text { Mean }\end{array}$ & $\begin{array}{l}\text { Unstable, } \\
\text { Above } \\
\text { Population } \\
\text { Mean }\end{array}$ & $\begin{array}{l}\text { stable, } \\
\text { Above } \\
\text { Population } \\
\text { Mean }\end{array}$ & $\begin{array}{l}\text { Unstable, } \\
\text { Above } \\
\text { Population } \\
\text { Mean }\end{array}$ & $\begin{array}{l}\text { Unstable, } \\
\text { Above } \\
\text { Population } \\
\text { Mean }\end{array}$ & $\begin{array}{l}\text { stable, } \\
\text { Above } \\
\text { Population } \\
\text { Mean }\end{array}$ \\
\hline 5. PusaSawani & $\begin{array}{l}\text { Unstable, } \\
\text { Above } \\
\text { Population } \\
\text { Mean }\end{array}$ & $\begin{array}{l}\text { stable, } \\
\text { Above } \\
\text { Population } \\
\text { Mean }\end{array}$ & $\begin{array}{l}\text { Unstable, } \\
\text { Above } \\
\text { Population } \\
\text { Mean }\end{array}$ & $\begin{array}{l}\text { Unstable, } \\
\text { Above } \\
\text { Population } \\
\text { Mean }\end{array}$ & $\begin{array}{l}\text { Unstable, } \\
\text { Above } \\
\text { Population } \\
\text { Mean }\end{array}$ & $\begin{array}{c}\text { Stable, } \\
\text { Above } \\
\text { Population } \\
\text { Mean }\end{array}$ \\
\hline
\end{tabular}

Eight genotypes exhibited high yield per plant than population mean but only five genotypes namely HRB-9-2, Pb-57, HOE-202, D-1-87-5 and Pusa Sawani exhibited the average response (bi near to zero), thus they were rated as highly stable genotypes under all the environments (Table 7). An observation of stability parameters showed that eight genotypes namely Sel.-10, B.O.-2, Pb-57, Vaishali, Vadhu, HRB-55, N.D.O.-25, HOE202 and Sel.-7 exhibited average fruit diameters above the population mean, average response and were found to be highly stable over all the environments as they were also associated with non-significant deviation from regression (Table 7).

AROH-1 and Sel.-2 exhibited higher fruit weight than the population mean, average response and the low value of deviation from regression indicating uniform performance by the over a wide range of environments in respect of this trait.
List of genotypes with high yield performance and high stability along with the stability of different quantitative characters under study is presented in Table 8. The genotypes HRB-92, Pb-57, HOE-202, D-1-87-5 and Pusa Sawani had average response and are highly stable for fruit yield per plant.

In conclusion, the results thus indicated that genotypes HRB-9-2, Pb-57, HOE-202, D-187-5, Pusa Sawani, 71-14, KS-312 and D-187-16 had higher potentialities over environments for producing high yield. The genotypes HRB-9-2, Pb-57, HOE-202, D-187-5 and Pusa Sawani had average response and are highly stable for fruit yield per plant. These genotypes are likely to perform well in all the environments of both the seasons (Spring-Summer and Kharif season). The results also indicated that the high yielding genotypes with high stability can be identified with appropriate testing in wide ranging environments. Thus genotypes as identified in the present study can further be exploited for 
higher yield and also in breeding for superior and stable genotypes of Bhindi.

\section{Acknowledgement}

Authors thank the Head of the Department of Horticulture (Vegetables \& Floriculture), Bihar Agriculture College, Bihar Agricultural University, Sabour, Bhagalpur for all the supports during the conduct of experiments.

\section{References}

Allard, R. W. and Bradshaw, A. D. (1964). Implications of Genotype-Environmental Interactions in Applied Plant Breeding. Crop Science, 4: 503-508

Burton, G.W. (1952). Quantitative inheritance in grasses. Proc. VI. Institute Grassland Congr., 1:155-157

Comstock, R.E.; Robinson, H.F. (1952). Estimation of average dominance of genes. In: Heterosis, Iowa State College Press Ch. 30, pp. 494-516.

Eberhart, S.A. and Russell, W.A. (1966). Stability parameters for comparing varieties. Crop. Sci., 6: 26-40.

FAOSTAT. 2014. Production - Crops data. Food and Agriculture Organization of the United Nations. http://www.fao.org/ faostat.

Javia R.M. 2014. Stability analysis for fruit yield and its attributing characters in okra [Abelmoschus esculentus (L.) Moench]. Int. J. Plant Sci. 9(1): 35 - 39.

Jensen, N.F. (1952). Intra-varietal diversification in oat breeding. Agron. J. 44: 30-34.

Jindal S.K., Arora D. and Ghai T.R. 2008. Stability analysis for earliness in okra
(Abelmoschus esculentus (L.) Moench), J. Res. Punjab Agric. Univ. 45(3 \& 4): 148 155.

Kachhadia V. H., Dangaria C.J., Vachhani J.H., Jivani L.L. and Shekhat H.G. 2011. Satbility analysis in okra (Abelmoschus esculentus L. Moench). Int. J. Plant Sci. 6(1): 34 - 39.

More, S. J., Chaudhari, K.N., Vaidya, G.B. and Chawla, S.L. (2018). Genotype x Environment interaction and stability analysis for earliness, seed yield and fruit yield in okra using the additive main effect and multiplicative interaction (AMMI). Int. J. Curr. Microbiol. App. Sci (2018) 7(3): 373-383.

Panse, V. G. and Sukhatme, P. V. (1985). Statistical Methods for Agricultural Workers, ICAR Publication, New Delhi, pp. 327-340.

Patil S.S., Desai D.T., Patil P.P. and Sunayan R. 2017. Genotype x environment interaction for fruit yield and component characters in okra [Abelmoschus esculentus (L.) Moench]. Electron. J. Plant Breed. 8(3): 787-791.

Poshiya, V.K. and Vashi, P.S., (1997), "Phenotypic stability of hybrids and their parents for fruit yield in okra (Abelmoschus esculentus L. Moench.)". Indian J. Genet. Plant Breed. 57(3): 266-268.

Sharma, R.K., Singh, R.S. and Kumar, A. (2016). Correlating fruit yield with important attributing traits Abelmoschus esculentus (L.). J. Biotech. Crop Sci., 5 (7): 88-94.

Sharma, R.K., Singh, R.S. and Kumar, A. (2017). Genetic variability, heritability and genetic advance Abelmoschus esculentus (L.). J. Biotech. Crop Sci., 6 (8): 77-85.

\section{How to cite this article:}

Ramesh K. Sharma, Ravi S. Singh, Arun Kumar, Ashok K. Singh, and Choudhary, S.K. 2019. Genotype X Environment Interaction and Stability Parameters of Genotypes for Different Traits in Bhindi [Abelmoschus esculentus (L.) Moench]. Int.J.Curr.Microbiol.App.Sci. 8(06): 3300-3310. doi: https://doi.org/10.20546/ijcmas.2019.806.393 